\title{
Impact of atrial fibrillation pattern on outcomes after left atrial appendage closure: lessons from the prospective LAARGE registry
}

\author{
Shinwan Kany ${ }^{1}$. Johannes Brachmann ${ }^{2}$. Thorsten Lewalter ${ }^{3} \cdot$ Ibrahim Akin $^{4} \cdot$ Horst Sievert $^{5} \cdot$ Uwe Zeymer $^{6}$. \\ Jakob Ledwoch ${ }^{7} \cdot$ Hüseyin Ince ${ }^{8} \cdot$ Dierk Thomas $^{9} \cdot$ Matthias Hochadel $^{10} \cdot$ Jochen Senges $^{10} \cdot$ Paulus Kirchhof $^{1}$. \\ Andreas Rillig ${ }^{1}$
}

Received: 12 January 2021 / Accepted: 17 May 2021 / Published online: 27 May 2021

(c) The Author(s) 2021

\begin{abstract}
Background Non-paroxysmal (NPAF) forms of atrial fibrillation (AF) have been reported to be associated with an increased risk for systemic embolism or death.

Methods Comparison of procedural details and long-term outcomes in patients (pts) with paroxysmal AF (PAF) against controls with NPAF in the prospective, multicentre observational registry of patients undergoing LAAC (LAARGE).

Results A total of 638 pts (PAF 274 pts, NPAF 364 pts) were enrolled. In both groups, a history of PVI was rare (4.0\% vs $1.6 \%, p=0.066)$. The total $\mathrm{CHA}_{2} \mathrm{DS}_{2}$-VASc score was lower in the PAF group $(4.4 \pm 1.5$ vs $4.6 \pm 1.5, p=0.033)$, while HASBLED score ( $3.8 \pm 1.1$ vs $3.9 \pm 1.1, p=0.40)$ was comparable. The rate of successful implantation was equally high $(97.4 \%$ vs $97.8 \%, p=0.77)$. In the three-month echo follow-up, LA thrombi $(2.1 \%$ vs $7.3 \%, p=0.12)$ and peridevice leak $>5 \mathrm{~mm}$ $(0.0 \%$ vs $7.1 \%, p=0.53)$ were numerically higher in the NPAF group. Overall, in-hospital complications occurred in $15.0 \%$ of the PAF cohort and $10.7 \%$ of the NPAF cohort $(p=0.12)$. In the one-year follow-up, unadjusted mortality (8.4\% vs $14.0 \%$, $p=0.039)$ and combined outcome of death, stroke and systemic embolism (8.8\% vs $15.1 \%, p=0.022)$ were significantly higher in the NPAF cohort. After adjusting for $\mathrm{CHA}_{2} \mathrm{DS}_{2}$-VASc and previous bleeding, NPAF was associated with increased death/stroke/systemic embolism (HR 1.67, 95\% CI 1.02-2.72, $p=0.041$ ).
\end{abstract}

Andreas Rillig

a.rillig@uke.de

1 Department of Cardiology, University Heart and Vascular Center Hamburg-Eppendorf, Martinistraße 52,

20251 Hamburg, Germany

2 Department of Cardiology, Angiology and Pneumology, Coburg Hospital, Coburg, Germany

3 Department of Medicine-Cardiology and Intensive Care, Hospital Munich-Thalkirchen, Munich, Germany

4 Department of Cardiology, University Hospital Mannheim, Mannheim, Germany

5 Cardio Vascular Centre, Frankfurt, Frankfurt/Main, Germany

6 Department of Cardiology, Ludwigshafen Hospital, Ludwigshafen, Germany

7 Department of Cardiology, Hospital Neuperlach, Munich, Germany

8 Department of Cardiology, University Hospital Rostock, Rostock, Germany

9 Department of Cardiology, University Hospital Heidelberg, Heidelberg, Germany

10 Stiftung Für Herzinfarktforschung (IHF), Ludwigshafen, Germany 
Conclusion Atrial fibrillation type did not impair periprocedural safety or in-hospital MACE patients undergoing LAAC. However, after one year, NPAF was associated with higher mortality.

\section{Graphic abstract}

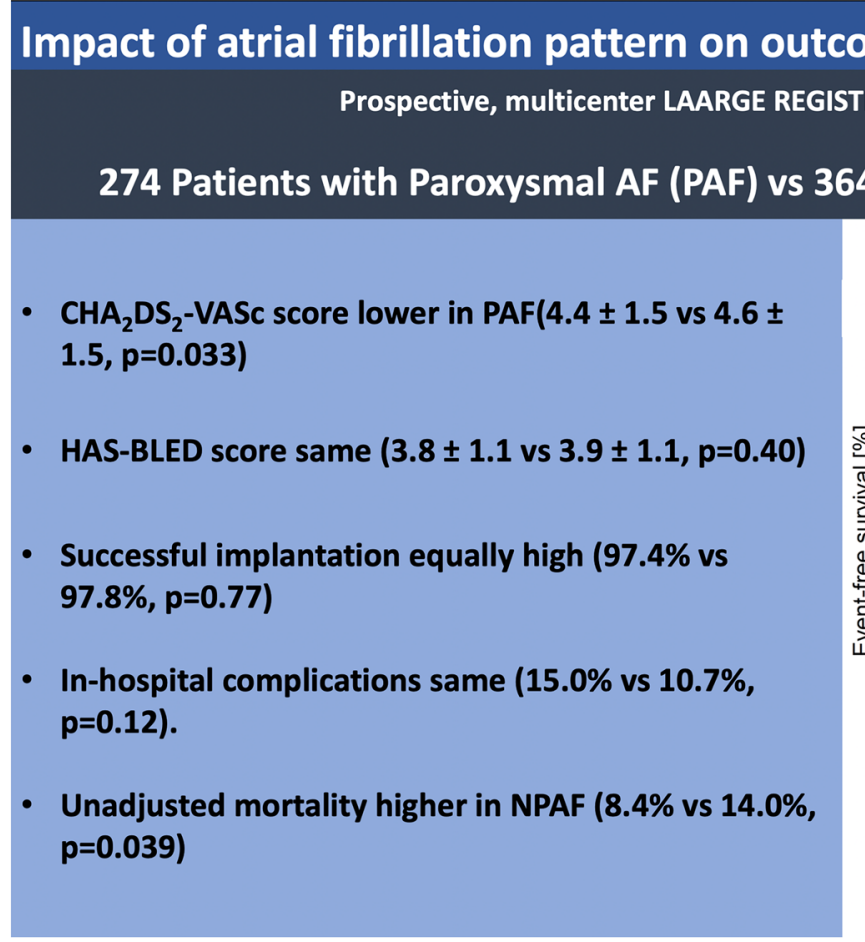

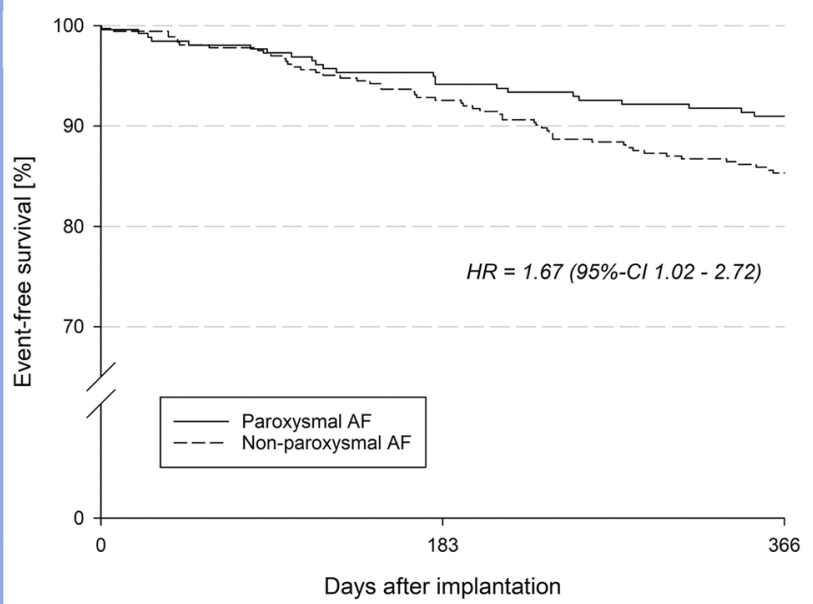

Composite of Death, Stroke and Systemic Embolism

Keywords Atrial fibrillation $\cdot$ AF type $\cdot$ Non-paroxysmal AF $\cdot$ Safety outcomes $\cdot$ Left atrial appendage closure

$\begin{array}{ll}\text { Abbreviations } \\ \text { AF } & \text { Atrial fibrillation } \\ \text { CA } & \text { Catheter ablation } \\ \text { DRT } & \text { Device-related thrombus } \\ \text { LAA } & \text { Left atrial appendage } \\ \text { LAAC } & \text { Left atrial appendage closure } \\ \text { NPAF } & \text { Non-paroxysmal atrial fibrillation } \\ \text { OAC } & \text { Oral anticoagulation } \\ \text { PAF } & \text { Paroxysmal atrial fibrillation }\end{array}$

\section{Introduction}

Atrial fibrillation (AF) is the most common arrhythmia with more than 33 million people affected worldwide [6]. The risk for stroke is increased fivefold and AF is believed to cause up to $23.5 \%$ of all strokes in patients over 80 [22, 25]. AF is a progressive disease with episodes $<7$ days referred to as paroxysmal $\mathrm{AF}$ (PAF), episodes $>7$ days as persistent $\mathrm{AF}$ and $>1$ year as long-standing persistent $\mathrm{AF}$, while permanent $\mathrm{AF}$ is defined as $\mathrm{AF}$ without further attempts to restore sinus rhythm according to patient's and physician's agreement [15].
Left atrial appendage closure (LAAC) is recommended in patients with contraindications to oral anticoagulation (OAC) and significant stroke risk [26]. The identification of patients who benefit from LAAC still remains challenging. In existing randomized controlled trials, LAAC was non-inferior to OAC in preventing strokes, but associated with significantly less major bleeding [20]. Recently, investigations from several trials and meta-analysis provided evidence that non-paroxysmal AF forms are associated with increased stroke risk and mortality compared with paroxysmal $\mathrm{AF}$, even with anticoagulation [11].

The prospective, real world Left Atrial Appendage Occluder Registry Germany (LAARGE) is a non-randomized and independently funded registry of LAAC in Germany. This study aims to investigate if paroxysmal vs non-paroxysmal forms of AF influence safety and long-term outcomes of LAAC. 


\section{Methods}

\section{Registry structure and data collection}

The multicenter LAARGE registry is a prospective, non-randomized study with 38 participating centers. Patients were enrolled from July 2014 to December 2015. The registry is managed by the non-profit organization "Institut für Herzinfarktforschung" (IHF, Ludwigshafen, Germany). There was no funding by industry. Selection of closure devices were left at the operator's discretion. Written informed consent was obtained from all patients. The privacy measures and data collection have been described previously [3]. Briefly, a web-based electronic case report form was used to collect baseline and procedural data with in-built check for plausibility. IHF conducted the one-year follow-up by reports from the implanting center and via a standardized phone interview. The study was carried out according to the declaration of Helsinki and approved by the ethics committee of the State Chamber of Medicine in Rhineland-Palatinate, Germany (837.173.14 (9412-F), 25.06.2014).

\section{Procedure and outcomes}

The detailed procedural methods have been described previously [9]. Patient selection was conducted according to best medical practice and the most recent guidelines and recommendations. The definitions of paroxysmal AF and non-paroxysmal AF were in accordance with the most recent guidelines [15]. The local implanting center was in charge of procedural protocol, device selection and antithrombotic regime. No limits to device or medication were given by the study protocol. Procedures were carried out in deep sedation using propofol or general anesthesia. Implantation success was defined as a stable position of the device according to the Munich consensus document [24]. Mortality, combined outcome of death and stroke as well as death, stroke and systemic embolism after one year were the primary outcomes. The outcome of death, stroke and systemic embolism was adjusted for $\mathrm{CHA}_{2} \mathrm{DS}_{2}$-VASc and previous bleeding.

\section{Statistical analysis}

Normally distributed continuous data and risk scores are given as means \pm standard deviation (SD), otherwise shown as medians with interquartile ranges (25th and 75 th percentiles). Categorical data are presented as relative percentages and absolute values. Statistical differences between both groups were compared using either Pearson's Chi-squared test or Mann-Whitney-Wilcoxon test. Fisher's exact test was used for rates of in-hospital and follow-up complications.
The 12-month event-rates of death, composite outcome of death and stroke, and composite of death, stroke and systemic embolism were calculated by the Kaplan-Meier method. Long-rank test was used to compare the outcomes between the groups. Hazard-ratios (HR) with 95\% confidence intervals (CI) were estimated using Cox regression. Adjustment of the composite outcome was done for $\mathrm{CHA}_{2} \mathrm{DS}_{2}$-VASc score and previous bleeding in a multiple Cox regression model and visualized by direct-adjusted survival curves computed in a stratified Cox model, where the expected survival curves are averaged over all patients in the analysis population. All statistical comparisons were two-sided, and $P$ values $<0.05$ were considered statistically significant. Analyses were performed using the Statistical Analysis System (SAS, Version 9.4, SAS Institute Inc., Cary, NC, USA).

\section{Results}

\section{Baseline characteristics}

A total of 638 patients were included in the analysis. The group with paroxysmal AF (PAF) included 274 patients, while the non-paroxysmal AF (NPAF) group included 364 patients (Table 1). The NPAF group consisted of patients with persistent $\mathrm{AF}(31.6 \%)$ and with longstanding persistent AF or permanent AF (68.4\%). Mean age (75.1 \pm 8.5 vs $76.6 \pm 7.3, p=0.099)$ and percentage of female patients (43.1\% vs $35.7 \%, P=0.059)$ were similar. Overall, a history of catheter ablation (CA) with pulmonary vein isolation was rare $(4.0 \%$ vs $1.6 \%, p=0.066)$. The PAF group had significantly less history of congestive heart failure $(19.0 \%$ vs $33.0 \%, p<0.001)$ while the left ventricular ejection fraction (LVEF) (median $60 \%$ vs $60 \%$ ) and the percentage of patients with reduced ejection fraction $(\mathrm{LVEF}<40 \%)$ were similar. Median heart rate was significantly higher in the NPAF cohort, but still adequately rate controlled.

The $\mathrm{CHA}_{2} \mathrm{DS}_{2}$-VASc score was lower in the PAF group $(4.4 \pm 1.5$ vs $4.6 \pm 1.5, p=0.033)$ but the percentage of patients with a $\mathrm{CHA}_{2} \mathrm{DS}_{2}$-VASc score $>2$ was statistically similar $(88.0 \%$ vs $91.2 \%, p=0.18)$. There was no difference in the HAS-BLED score $(3.8 \pm 1.1$ vs $3.9 \pm 1.1, p=0.40)$. An overview of indications for LAAC is given in the supplemental Table 1.

\section{Left atrial appendage anatomy and procedural data}

The LA diameter (median $46 \mathrm{~mm}$ vs $49 \mathrm{~mm}, p<0.001$ ) and LA volume $\left(20.5 \mathrm{~cm}^{2}\right.$ vs $\left.29.0 \mathrm{~cm}^{2}, p=0.001\right)$ were significantly smaller in the PAF cohort compared to the NPAF cohort (supplemental Table 2). The prevalence of LAA thrombus $(0.4 \%$ vs $0.9 \%, p=0.50)$ and LAA sludge 
Table 1 Baseline Characteristics of patients with paroxysmal and non-paroxysmal AF

\begin{tabular}{|c|c|c|c|c|}
\hline & $\begin{array}{l}\text { Paroxysmal AF cohort } \\
(n=247)\end{array}$ & $\begin{array}{l}\text { Non-paroxysmal AF cohort } \\
(n=364)\end{array}$ & $P$ value & Odds ratio $(95 \% \mathrm{CI})$ \\
\hline Number of patients & $247(42.9 \%)$ & $364(57.1 \%)$ & & - \\
\hline Age, years & $75.1 \pm 8.5$ & $76.6 \pm 7.3$ & 0.099 & - \\
\hline Female, $\%$ & 43.1 & 35.7 & 0.059 & - \\
\hline Height, $\mathrm{cm}$ & $170(163,175)$ & $172(165,177)$ & 0.014 & - \\
\hline Weight, kg & $79(69,86)$ & $80(70,90)$ & 0.030 & - \\
\hline \multicolumn{5}{|l|}{ History of AF } \\
\hline Paroxysmal AF, \% & 100.0 & 0.0 & & - \\
\hline Persistent AF, \% & 0.0 & 31.6 & & - \\
\hline Permanent or LSP AF, \% & 0.0 & 68.4 & & - \\
\hline History of PVI, \% & 4.0 & 1.6 & 0.066 & $2.50(0.91-6.83)$ \\
\hline \multicolumn{5}{|l|}{ Cardiac history } \\
\hline Coronary artery disease, $\%$ & 45.3 & 46.2 & 0.82 & $0.96(0.70-1.32)$ \\
\hline History of MI, \% & 10.9 & 9.1 & 0.43 & $1.23(0.73-2.08)$ \\
\hline Valvular heart disease, $\%$ & 20.4 & 20.9 & 0.89 & $0.97(0.66-1.43)$ \\
\hline Cardiomyopathy, \% & 6.9 & 7.1 & 0.92 & $0.97(0.52-1.79)$ \\
\hline History of congestive heart failure, $\%$ & 19.0 & 33.0 & $<0.001$ & $0.48(0.33-0.69)$ \\
\hline LVEF, \% (median) & $60(50,60)$ & $60(50,60)$ & 0.26 & - \\
\hline $\mathrm{LVEF}<40 \%, \%$ & 12.6 & 11.5 & 0.65 & \\
\hline Heart rate $($ median $) / \min$ & $70(62,78)$ & $76(67,83)$ & $<0.001$ & \\
\hline Hypertension, \% & 93.1 & 92.9 & 0.92 & $0.62(0.43-0.89)$ \\
\hline No structural heart disease, $\%$ & 23.7 & 17.3 & 0.045 & $1.03(0.56-1.91)$ \\
\hline \multicolumn{5}{|l|}{ Extracardiac history } \\
\hline Diabetes mellitus, $\%$ & 31.8 & 35.7 & 0.30 & $0.84(0.60-1.17)$ \\
\hline Chronic kidney disease, $\%$ & 33.9 & 40.7 & 0.083 & $0.75(0.54-1.04)$ \\
\hline Vascular disease (e.g., PAD), $\%$ & 28.1 & 24.7 & 0.34 & $1.19(0.83-1.70)$ \\
\hline Chronic liver disease, $\%$ & 8.0 & 11.0 & 0.21 & $0.71(0.41-1.22)$ \\
\hline Alcohol use disorder, $\%$ & 2.6 & 5.0 & 0.13 & $0.51(0.21-1.23)$ \\
\hline \multicolumn{5}{|l|}{ Risk scores } \\
\hline CHA2DS2-VASc Score & $4.4 \pm 1.5$ & $4.6 \pm 1.5$ & 0.033 & - \\
\hline CHA2DS2-VASc Score > 2, $\%$ & 88.0 & 91.2 & 0.18 & $0.70(0.42-1.18)$ \\
\hline HAS-BLED Score & $3.8 \pm 1.1$ & $3.9 \pm 1.1$ & 0.40 & - \\
\hline Stroke or transient sichemic attack & $27.0 \%$ & $27.2 \%$ & 0.96 & - \\
\hline
\end{tabular}

Patients with paroxysmal AF have less heart failure and thus lower $\mathrm{CHA}_{2} \mathrm{DS}_{2}$-VASc scores with similar HAS-BLED scores compared with patients with non-paroxsmal $\mathrm{AF}$

$A F$ atrial fibrillation, $C I$ confidence interval, $L S P$ long-standing persistent, $P V I$ pulmonary vein isolation, $M I$ myocardial infarction, $L V E F$ left ventricular ejection fraction, $P A D$ peripheral artery disease; displayed are percentages and numbers or median and quartiles; $P$ values $<0.05$ are considered significant, tested with either Pearson's chi-squared test or Mann-Whitney-Wilcoxon test

(14.1\% vs $15.3 \%, p=0.70)$ was similar in both groups. Acute implantation success of LAAC devices was equally high $(97.4 \%$ vs $97.8 \%, p=0.77$ ) in both groups (Table 2). The PAF cohort presented significantly more often in sinus rhythm $(69.0 \%$ vs $2.2 \%, p<0.001)$ and significantly less in AF $(23.7 \%$ vs $92.9 \%, p<0.001)$. Most procedures were done under conscious sedation $(85.0 \%$ vs $83.5 \%, p=0.62)$, followed by general anesthesia $(10.6 \%$ vs $12.1 \%, p=0.57)$. There was no difference in device selection. The number of device-retractions and repositioning $(1.6 \pm 1.3$ vs $1.7 \pm 1.2$, $p=0.069$ ) was comparable in both groups but skewing towards the NPAF group. The PAF cohort had significantly longer procedural (61 $\mathrm{min}$ vs $55 \mathrm{~min}, p=0.004$ ) and fluoroscopy (11 $\mathrm{min}$ vs $9 \mathrm{~min}, p=0.002$ ) times. There was no significant difference in peridevice leak $(4.6 \%$ vs $5.6 \%$, $p=0.57)$ or left-to-right shunts $(6.7 \%$ vs $4.5 \%, p=0.22)$ after the procedure. 
Table 2 Procedural data

\begin{tabular}{|c|c|c|c|c|}
\hline & $\begin{array}{l}\text { Paroxysmal AF } \\
\text { cohort }(n=274)\end{array}$ & $\begin{array}{l}\text { Non-paroxysmal } \\
\text { AF cohort } \\
(n=364)\end{array}$ & $P$ value & Odds ratio $(95 \% \mathrm{CI})$ \\
\hline Implant success, $\%$ & 97.4 & 97.8 & 0.77 & $0.86(0.31-2.39)$ \\
\hline \multicolumn{5}{|l|}{ Rhythm at implant } \\
\hline Sinus rhythm, \% & 69.0 & 2.2 & $<0.001$ & $98.95(46.93-208.63)$ \\
\hline Atrial fibrillation, $\%$ & 23.7 & 92.9 & $<0.001$ & $0.02(0.01-0.04)$ \\
\hline Pacing, $\%$ & 8.4 & 5.8 & 0.20 & $1.50(0.81-2.76)$ \\
\hline \multicolumn{5}{|l|}{ Anesthesia } \\
\hline Conscious sedation, $\%$ & 85.0 & 83.5 & 0.62 & $1.12(0.72-1.72)$ \\
\hline General anesthesia, $\%$ & 10.6 & 12.1 & 0.57 & $0.86(0.53-1.42)$ \\
\hline \multicolumn{5}{|l|}{ LAAC device } \\
\hline Watchman, \% & 45.3 & 42.4 & 0.48 & $1.12(0.82-1.54)$ \\
\hline Amplatzer Cardiac Plug, \% & 26.3 & 28.9 & 0.46 & $0.88(0.62-1.25)$ \\
\hline Amplatzer Amulet, \% & 25.5 & 25.6 & 0.98 & $1.00(0.70-1.43)$ \\
\hline Other device*, $\%$ & 2.9 & 3.0 & 0.94 & $0.96(0.38-2.43)$ \\
\hline \multicolumn{5}{|l|}{ Periprocedural data } \\
\hline Sheath retractions & $1.6 \pm 1.3, N=263$ & $1.7 \pm 1.2, N=356$ & 0.069 & - \\
\hline Duration, min & $61(46,85)$ & $55(42,74)$ & 0.004 & - \\
\hline Fluroroscopy duration, min & $11(8,16)$ & $9(7,14)$ & 0.002 & - \\
\hline Dose area product, $\mathrm{cGy} * \mathrm{~cm}^{2}$ & $1999(851,4010)$ & $2091(856,4512)$ & 0.84 & - \\
\hline Device dislodgment, $\%$ & $1.8(5)$ & $1.1(4)$ & 0.44 & $1.67(0.44-6.27)$ \\
\hline Catheter-based retrieval, $\%$ & $5 / 5$ & $4 / 4$ & & -- \\
\hline Surgical retrieval, $\%$ & $0 / 5$ & $0 / 4$ & & -- \\
\hline Peridevice leak, $\%$ & $4.6(12)$ & $5.6(20)$ & 0.57 & $0.81(0.39-1.68)$ \\
\hline$<3 \mathrm{~mm}$ & $8 / 12$ & $16 / 20$ & & $0.50(0.10-2.54)$ \\
\hline $3-5 \mathrm{~mm}$ & $4 / 12$ & $4 / 20$ & & $2.00(0.39-10.16)$ \\
\hline$>5 \mathrm{~mm}$ & $0 / 12$ & $0 / 20$ & & -- \\
\hline Left-right shunt, $\%$ & 6.7 & 4.5 & 0.22 & $1.53(0.77-3.06)$ \\
\hline
\end{tabular}

$A F$ atrial fibrillation, $C I$ confidence interval, $L A A C$ left atrial appendage closure; displayed are percentages and numbers or median and quartiles

*Other devices include Occlutech, LAmbre and LARIAT; $P$ values $<0.05$ are considered significant, tested with Fisher`s exact test

\section{In-hospital safety data}

Incidences of MACCE (Death, Stroke or MI) were equally rare $(0.7 \%$ vs $0.3 \%, p=0.58)$ in both groups (Table 3$)$. Other severe complications $(4.7 \%$ vs $3.6 \%, p=0.55)$, including severe bleeding and AV-Fistula, were similar. Moderate complications were similar in both groups $(10.9 \%$ vs $8.8 \%$, $p=0.42)$ as well as minor complications $(2.9 \%$ vs $2.5 \%$, $p=0.81$ ). The overall incidence of combined severe and moderate complications was numerically higher in the PAF cohort $(15.0 \%$ vs $10.7 \%, p=0.12)$.

\section{Antithrombotic therapy}

Before the procedure, therapeutic anticoagulation was comparable in both groups $(47.1 \%$ vs $50.8 \%, p=0.35)$. Single antiplatelet therapy (SAPT) was significantly higher in the
PAF cohort compared with the NPAF cohort $(21.2 \%$ vs $11.8 \%, p=0.001$ ). At discharge, dual antiplatelet therapy (DAPT) was the main antithrombotic therapy in both groups ( $83.1 \%$ vs $85.2 \%, p=0.48$ ). There were no significant differences in the antithrombotic therapy at discharge or at the one-year follow-up (details in Table 4).

\section{Follow-up safety data}

In the echocardiographic FU after ca. 100 days (103d (47d, 194d) vs 97d (54d, 186d)), LA thrombi $(2.1 \%$ vs $7.3 \%, p=0.12)$ and peridevice leak $>5 \mathrm{~mm}(0.0 \%$ vs $7.1 \%$, $p=0.53$ ) were numerically higher in the NPAF group. Overall device dislodgment was rare $(2.2 \%$ vs $2.5 \%, p=1.00)$. Groin complications $(2.6 \%$ vs $3.9 \%, p=0.50)$ were rare in both cohorts. Pericardial effusion was numerically higher in the PAF cohort $(6.2 \%$ vs $3.3 \%, p=0.09$, OR 1.94 (0.91-4.14)). There was no difference in stroke $(0.4 \%$ vs 
Table 3 In-hospital safety data after the procedure

\begin{tabular}{|c|c|c|c|c|}
\hline & $\begin{array}{l}\text { Paroxysmal AF } \\
\text { cohort }(n=274)\end{array}$ & $\begin{array}{l}\text { Non-paroxysmal AF } \\
\text { cohort }(n=364)\end{array}$ & $P$ value & Odds ratio $(95 \% \mathrm{CI})$ \\
\hline MACCE (death, MI, stroke), \% & 0.7 & 0.3 & 0.58 & \\
\hline Death, $\%$ & 0.7 & 0.0 & 0.18 & - \\
\hline MI, \% & 0.0 & 0.3 & 1.00 & - \\
\hline Stroke, $\%$ & 0.0 & 0.3 & 1.00 & - \\
\hline Other severe complications, $\%$ & 4.7 & 3.6 & 0.55 & $1.34(0.61-2.95)$ \\
\hline Severe bleeding, $\%$ & 1.1 & 1.1 & 1.00 & $1.00(0.22-4.49)$ \\
\hline AV-Fistula/Aneurysmal hematoma, $\%$ & 1.1 & 0.8 & 1.00 & $1.33(0.27-6.65)$ \\
\hline Pericardial effusion—surgical treatment, $\%$ & 0.7 & 0.0 & 0.18 & - \\
\hline Pericardial effusion-interventional treatment, $\%$ & 2.2 & 1.9 & 1.00 & $1.14(0.38-3.44)$ \\
\hline Hemo-/pneumothorax-surgical treatment, $\%$ & 0.0 & 0.0 & - & - \\
\hline Device dislodgment—surgical treatment, $\%$ & 0.0 & 0.0 & - & - \\
\hline Device dislodgment-interventional treatment, $\%$ & 0.4 & 0.3 & 1.00 & $1.33(0.08-21.29)$ \\
\hline MACCE + other severe complication, $\%$ & 5.5 & 3.8 & 0.34 & $1.45(0.69-3.05)$ \\
\hline Moderate complications, $\%$ & 10.9 & 8.8 & 0.42 & $1.28(0.75-2.16)$ \\
\hline TIA, \% & 0.0 & 0.0 & - & - \\
\hline Non-fatal CPR, \% & 0.4 & 0.5 & 1.00 & $0.66(0.06-7.35)$ \\
\hline Moderate bleeding, $\%$ & 1.8 & 1.9 & 1.00 & $0.95(0.30-3.02)$ \\
\hline Access site infection, $\%$ & 0.4 & 0.0 & 0.43 & -- \\
\hline Groin hematoma, $\%$ & 2.2 & 3.3 & 0.48 & $0.66(0.24-1.77)$ \\
\hline Pericardial effusion-conservative treatment, $\%$ & 2.9 & 0.8 & 0.063 & $3.62(0.95-13.77)$ \\
\hline Hemo-/pneumothorax-interventional treatment, $\%$ & 0.4 & 0.3 & 1.00 & $1.33(0.08-21.35)$ \\
\hline Hemo-/pneumothorax-conservative treatment, $\%$ & 0.0 & 0.0 & - & - \\
\hline Device dislodgment—at index procedure, $\%$ & 1.5 & 0.8 & 0.47 & $1.78(0.39-8.01)$ \\
\hline Minor complications, $\%$ & 2.9 & 2.5 & 0.81 & $1.19(0.45-3.12)$ \\
\hline Overall complications (severe and moderate), $\%$ & 15.0 & 10.7 & 0.12 & $1.47(0.92-2.35)$ \\
\hline
\end{tabular}

The AF form does influence safety measures

$A F$ atrial fibrillation, $M I$ myocardial infarction, $A V$ arteriovenous, $T I A$ transient ischemic attack, $C P R$ cardiopulmonary resuscitation; displayed are percentages and numbers; $P$ values $<0.05$ are considered significant, tested with tested with Fisher's exact test

$1.4 \%, p=0.24)$ or TIA $(0.4 \%$ vs $0.3 \%, p=1.0)$, however, MI occurred significantly less often $(0.0 \%$ vs $1.7 \%, p=0.04)$ in the PAF cohort compared with the NPAF cohort. In the unadjusted outcomes, the PAF cohort demonstrated significantly lower all-cause mortality $(8.4 \%$ vs $14.0 \%, p=0.039)$, combined death and stroke $(8.8 \%$ vs $14.8 \%, p=0.028)$ and combined outcome of death, stroke and systemic embo$\operatorname{lism}(8.8 \%$ vs $15.1 \%, p=0.022)$ (Table 5). In the adjusted $\left(\mathrm{CHA}_{2} \mathrm{DS}_{2}\right.$-VASc and previous bleeding) outcome of eventfree survival NPAF was associated with increased composite outcome of death/stroke/systemic embolism (HR 1.67, 95\% CI 1.02-2.72, $p=0.041$ ) (Fig. 1).

\section{Discussion}

Among patients undergoing LAAC, non-paroxysmal forms of AF are associated with higher rates of death, stroke or systemic embolism after one year in a prospective real-world setting regardless of the $\mathrm{CHA}_{2} \mathrm{DS}_{2}$-VASc score. Complications are comparable between patients with paroxysmal or non-paroxysmal AF, though procedure and fluoroscopy times are longer in the former.

Recently, a case series showed that LAAC is associated with acute brain lesion in MRI of unknown clinical relevance [21]. The impact of AF type on stroke risk has long been debated. A post-hoc analysis of the ACTIVE-W trial found no association of AF type on thromboembolic events. However, differences in anticoagulation between PAF and persistent $\mathrm{AF}$ and low $\mathrm{CHADS}_{2}$ scores were a major limitation [16]. In a post-hoc analysis of the ROCKET-AF trial, persistent AF was significantly associated with higher systemic embolism, stroke and all-cause mortality [23]. Recently, a meta-analysis of 12 studies, with almost 100.000 patient showed that NPAF was associated with a higher risk for thromboembolism and all-cause mortality, no difference in bleeding was observed [11]. Interestingly, sub-analyses of the ENTRUST-AF and ENSURE-AF trials showed an 
Table 4 Antithrombotic therapy

\begin{tabular}{|c|c|c|c|c|}
\hline & $\begin{array}{l}\text { Paroxysmal } \\
\text { AF cohort }\end{array}$ & $\begin{array}{l}\text { Non-paroxysmal } \\
\text { AF cohort }\end{array}$ & $P$ value & Odds ratio $(95 \% \mathrm{CI})$ \\
\hline Therapy at admission & $n=274$ & $n=364$ & & \\
\hline Anticoagulation, $\%$ & 47.1 & 50.8 & 0.35 & $0.86(0.63-1.18)$ \\
\hline DAPT, $\%$ & 7.7 & 6.9 & 0.70 & $1.13(0.62-2.06)$ \\
\hline SAPT, $\%$ & 21.2 & 11.8 & 0.001 & $2.00(1.30-3.08)$ \\
\hline Double antithrombotic therapy, $\%$ & 8.8 & 8.8 & 0.99 & $1.00(0.57-1.73)$ \\
\hline Triple antithrombotic therapy, $\%$ & 2.2 & 3.6 & 0.31 & $0.60(0.23-1.61)$ \\
\hline No antithrombotic therapy, $\%$ & 13.1 & 18.1 & 0.088 & $0.68(0.44-1.06)$ \\
\hline Therapy at discharge, $\%$ & $n=272$ & $n=364$ & & \\
\hline Anticoagulation, $\%$ & 3.3 & 2.5 & 0.53 & $1.35(0.53-3.45)$ \\
\hline DAPT, $\%$ & 83.1 & 85.2 & 0.48 & $0.86(0.56-1.31)$ \\
\hline SAPT, $\%$ & 4.0 & 1.9 & 0.11 & $2.15(0.82-5.62)$ \\
\hline Double antithrombotic therapy, $\%$ & 7.0 & 6.9 & 0.95 & $1.02(0.55-1.89)$ \\
\hline Triple antithrombotic therapy, $\%$ & 2.2 & 2.7 & 0.67 & $0.80(0.29-2.22)$ \\
\hline No antithrombotic therapy, $\%$ & 0.4 & 0.5 & 0.74 & $0.67(0.06-7.40)$ \\
\hline PPI, \% & 48.0 & 46.3 & 0.67 & $1.07(0.78-1.47)$ \\
\hline NSAID, $\%$ & 8.5 & 6.1 & 0.24 & $1.44(0.78-2.64)$ \\
\hline Therapy at one-year follow-up & $n=223$ & $n=284$ & & \\
\hline Anticoagulation, $\%$ & 5.4 & 4.2 & 0.54 & $1.29(0.57-2.93)$ \\
\hline DAPT, $\%$ & 6.3 & 6.7 & 0.85 & $0.93(0.46-1.91)$ \\
\hline SAPT, $\%$ & 78.9 & 73.6 & 0.16 & $1.34(0.89-2.04)$ \\
\hline Double antithrombotic therapy, \% & 0.9 & 1.8 & 0.41 & $0.50(0.10-2.63)$ \\
\hline Triple antithrombotic therapy, $\%$ & 0.0 & 0.7 & 0.21 & - \\
\hline No antithrombotic therapy, $\%$ & 8.5 & 13.0 & 0.11 & $0.62(0.35-1.11)$ \\
\hline PPI, \% & 43.5 & 39.4 & 0.36 & $1.18(0.83-1.69)$ \\
\hline NSAID, $\%$ & 5.4 & 4.9 & 0.82 & $1.10(0.50-2.42)$ \\
\hline
\end{tabular}

$O R$ odds ratio, $C I$ confidence interval, $D A P T$ dual antiplatelet therapy, SAPT single antiplatelet therapy, $P P I$ proton pump inhibitor, NSAID non-steroidal anti-inflammatory drugs; displayed are percentages and numbers; $P$ values $<0.05$ are considered significant, tested with either Pearson's chi-squared test or MannWhitney-Wilcoxon test

association of MI and PAF not observed in patients with persistent $\mathrm{AF}[13,14]$. The authors propose that PAF is associated with microcirculatory flow abnormalities and may increase events in vulnerable myocardium $[13,14]$. However, several limitations may limit the generalization of the findings. For Instance, patients with PAF in ENSURE-AF were significantly more likely to be without anticoagulation at baseline compared with NPAF patients ( $47 \%$ vs $23 \%$, $p<0.0001)$ and follow-up encompassed only 58 days [13, 14]. While the increased stroke risk and mortality for NPAF has been shown repeatedly, the influence of AF types on LAA thrombus formation and outcomes after LAAC has not been a focus of research [5].

Our cohort had comparable $\mathrm{CHA}_{2} \mathrm{DS}_{2}$-VASc scores (PAF: $4.4 \pm 1.5$ vs NPAF: $4.6 \pm 1.5$ ) to other registries such as the European EWOLUTION registry $(4.5 \pm 1.6)$. While the one-year mortality in EWOLUTION was 9.8\% and thus comparable to the PAF cohort (8.4\%), the NPAF cohort demonstrated a higher mortality of $14 \%$ [2]. Incidences of major bleeding as a surrogate for differences in major comorbidities were similar in EWOLUTION (2.6\%) and our cohort (2.8\%). Furthermore, CA and antiarrhythmic drug therapy have been reported to potentially decrease stroke risk and mortality in select patients [10,17]. Our study shows that the cohort undergoing LAAC is treated very rarely with CA ( $4.0 \%$ vs $1.6 \%)$. The influence of CA on the prevention of stroke in $\mathrm{AF}$ is still studied and may be of importance in the population undergoing LAAC [4, 19]. We also report a higher incidence of congestive heart failure in NPAF patients compared with PAF despite similar LVEF. One aspect that may serve as an explanation may be the rate control of atrial fibrillation. While we did observe a higher median heart rate with NPAF compared with PAF, the overall heart rate was still sufficiently rate controlled (median 76/min (67-83)). Certainly, heart failure with preserved ejection fraction may be a factor, considering the NPAF cohort were much more likely in AF than compared with PAF patients.

What is more, there is evidence that NPAF is associated with larger LAA volume as well as non-chicken-wing LAA 
Table 5 Follow-up safety data

\begin{tabular}{|c|c|c|c|c|}
\hline & Paroxysmal AF $(n=274)$ & $\begin{array}{l}\text { Non-paroxysmal AF } \\
(n=364)\end{array}$ & $P$ value & Odds ratio $(95 \% \mathrm{CI})$ \\
\hline Echocardiography FU documented, \% & $34.9(95 / 272)$ & $34.1(124 / 364)$ & 0.87 & - \\
\hline Days to echo FU & $103(47,194)$ & $97(54,186)$ & 0.94 & - \\
\hline Peridevice leak, $\%$ & $16.8(16 / 95)$ & $22.6(28 / 124)$ & 0.31 & $0.69(0.35-1.37)$ \\
\hline$<3 \mathrm{~mm}$ & $13 / 16$ & $22 / 28$ & 1.00 & $1.18(0.25-5.55)$ \\
\hline $3-5 \mathrm{~mm}$ & $3 / 16$ & $4 / 28$ & 0.69 & $1.38(0.27-7.15)$ \\
\hline$>5 \mathrm{~mm}$ & $0 / 16$ & $2 / 28$ & 0.53 & $0.28(0.06-1.31)$ \\
\hline LA thrombus, $\%$ & $2.1(2 / 94)$ & $7.3(9 / 123)$ & 0.12 & $0.28(0.06-1.31)$ \\
\hline LA sludge, $\%$ & $0.0(0 / 27)$ & $2.7(1 / 37)$ & 1.00 & - \\
\hline \multicolumn{5}{|l|}{ One year follow-up } \\
\hline One-year FU documented, \% & $97.1(266 / 274)$ & $98.1(357 / 364)$ & 0.44 & - \\
\hline Days to one-year FU & $377(367,402)$ & $380(367,411)$ & 0.50 & - \\
\hline Device dislodgment, $\%$ & $2.2(6)$ & $2.5(9)$ & 1.00 & $0.88(0.31-2.51)$ \\
\hline Surgical treatment & $0 / 6$ & $3 / 9$ & 0.23 & - \\
\hline Interventional treatment & $6 / 6$ & $4 / 9$ & 0.044 & - \\
\hline Conservative treatment & $0 / 6$ & $2 / 9$ & 0.49 & - \\
\hline Groin complications, $\%$ & $2.6(7)$ & $3.9(14)$ & 0.50 & $0.66(0.26-1.65)$ \\
\hline Surgical treatment & $1 / 7$ & $2 / 14$ & 1.00 & $1.00(0.07-13.37)$ \\
\hline Blood transfusion & $0 / 7$ & $0 / 14$ & - & - \\
\hline Conservative treatment & $6 / 7$ & $12 / 14$ & 1.00 & $1.00(0.07-13.37)$ \\
\hline Pericardial effusion, $\%$ & $6.2(16)$ & $3.3(12)$ & 0.087 & $1.94(0.91-4.14)$ \\
\hline Surgical treatment & $2 / 16$ & $0 / 12$ & 0.49 & - \\
\hline Interventional treatment & $7 / 16$ & $7 / 12$ & 0.70 & $0.56(0.12-2.53)$ \\
\hline Conservative treatment & $7 / 16$ & $5 / 12$ & 1.00 & $1.09(0.24-4.95)$ \\
\hline Stroke, $\%$ & 0.4 & 1.4 & 0.24 & $0.31(0.03-2.81)$ \\
\hline TIA, $\%$ & 0.4 & 0.3 & 1.00 & $0.26(0.03-2.27)$ \\
\hline MI, \% & 0.0 & 1.7 & 0.040 & $1.33(0.08-21.37)$ \\
\hline Bleeding (severe or moderate), $\%$ & 7.3 & $6.9 \%(25)$ & 0.88 & - \\
\hline Severe bleeding, $\%$ & 1.5 & 2.8 & 0.41 & $1.07(0.58-1.97)$ \\
\hline Moderate bleeding, $\%$ & 5.9 & 4.1 & 0.35 & $0.52(0.16-1.69)$ \\
\hline \multicolumn{5}{|l|}{ Composite outcomes $\dagger$} \\
\hline Mortality, \% & 8.4 & 14.0 & 0.039 & $0.59(0.36-0.98) \mathrm{HR}$ \\
\hline Death/stroke, $\%$ & 8.8 & 14.8 & 0.028 & $0.58(0.36-0.95) \mathrm{HR}$ \\
\hline Death/stroke/SE, \% & 8.8 & 15.1 & 0.022 & $0.57(0.35-0.93) \mathrm{HR}$ \\
\hline
\end{tabular}

$O R$ odds ratio, $C I$ confidence interval, TIA transient ischemic attack, $M I$ myocardial infarction, $S E$ systemic embolism, $K M$ Kaplan-Meier estimate, $L O$ Log-rank test, $H R$ hazard ratio; displayed are percentages and numbers; $P$ values $<0.05$ are considered significant, tested with either Fisher's exact test or Mann-Whitney-Wilcoxon test

${ }^{\dagger}$ Kaplan-Meier estimates at 1 year after the index procedure, compared by log-rank test

morphologies [18]. These morphologies are associated with a higher risk for stroke [7]. This is furthermore supported by a recent work showing that long standing persistent AF leads to larger LAA sizes, which require larger sizes of LAAC devices and lead to more residual leaks after closure [12]. Considering that most patients in the NPAF cohort were diagnosed with long-standing persistent or permanent AF, it is highly likely they have a longer history of AF compared with PAF patients.

While we could observe increasing volume of LA and LAA ostia in the NPAF cohort, this was not associated with more residual leaks in the follow-up. We observed longer fluoroscopy and procedure times in PAF patients, which might be explained by the higher rate of sinus rhythm during implant and thus potentially more challenging LAA movement.

Our data shows numerically higher incidences of LA thrombus in the NPAF cohort. In an analysis of 1739 patients in prospective trials or registries in the US receiving a Watchman device, DRT was significantly associated with large LAA diameter and permanent atrial fibrillation and higher risk of stroke [8]. Likewise, in the prospective 
Fig. 1 One-year outcomes of event-free survival (death/ stroke/systemic embolism) unadjusted $\mathbf{a}$ and adjusted for for $\mathrm{CHA}_{2} \mathrm{DS}_{2}$-VASc and previous bleeding $\mathbf{b}$ in patients with paroxysmal AF or nonparoxysmal AF; $P$ values $<0.05$ are considered significant, tested with either Pearson's chi-squared test or Mann-Whitney-Wilcoxon test
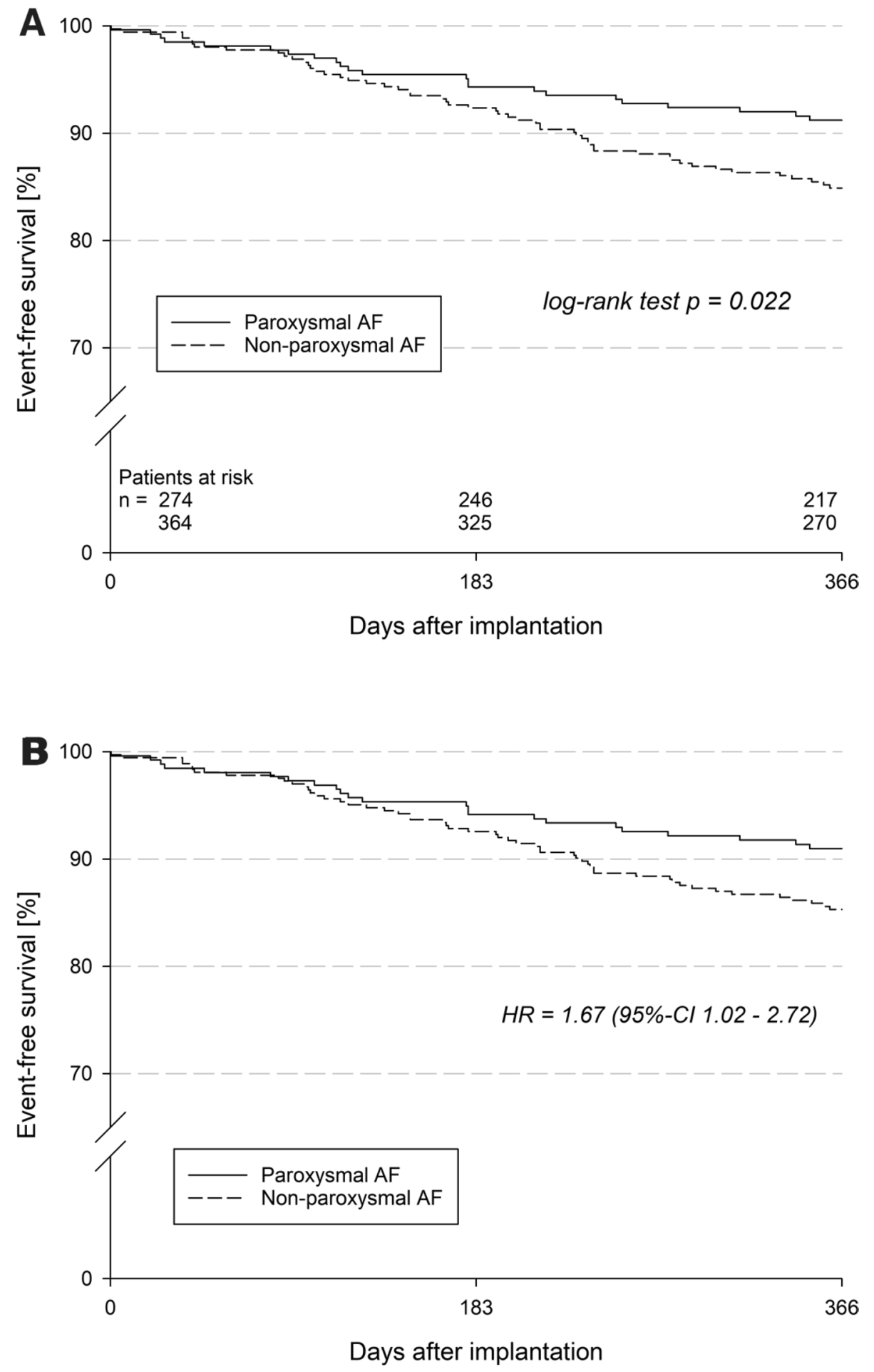

Amulet Observational study the incidence of DRT was reported with $1.7 \%$ with larger LAA orifice being a significant predictor for DRT and associated increased risk of stroke [1]. In line with these observations, in our cohort NPAF was associated with an increased risk for death, stroke or systemic embolism. Our observed findings remain after adjusting for $\mathrm{CHA}_{2} \mathrm{DS}_{2}$-VASc score, underlining the effect NPAF may have on outcomes after LAAC. Future studies are needed and, ultimately, randomized trials to evaluate different antithrombotic strategies after LAAC depending on AF patterns observed. 


\section{Limitations}

Confounding factors cannot be excluded due to the observational registry format. Additionally, there was no standardized process for patient screening, device selection and LAAC procedure.

There may have been significant differences between the operators' experience as well as the center volume of procedures. Also, increased experience and technical improvements may lead to more favorable outcomes in a contemporary study. Newer devices such as the Watchman FLX were not included in this study. Furthermore, we lack sufficient follow-up echocardiography data to report three-month and one-year incidences of DRT and peri-device leak. Centers were encouraged to enroll patients consecutively but not obligated; therefore, a selection bias cannot be excluded. There was no monitoring of AF burden or changes of $\mathrm{AF}$ pattern during follow-up but since most patients in the NPAF cohort were in longstanding persistent or permanent $\mathrm{AF}$, the AF burden is unlikely to have changed during follow-up.

Centers were also encouraged to report adverse events which may include a certain reporting bias.

\section{Conclusion}

Patients with non-paroxysmal forms of atrial fibrillation undergoing LAAC are more likely to have larger LA volume as well as larger LAA size and orifices. This did, however, not impair periprocedural safety or in-hospital MACE. After one year, NPAF was associated with higher mortality, stroke or systemic embolism.

Supplementary Information The online version contains supplementary material available at https://doi.org/10.1007/s00392-021-01874-3.

Funding Open Access funding enabled and organized by Projekt DEAL. The study was funded by an unrestricted grant from foundation 'Stiftung Institut für Herzinfarktforschung Ludwigshafen' (Ludwigshafen, Germany).

Availability of data and material All data and material available at IHF Ludwigshafen.

\section{Declarations}

Conflict of interest SK, JL: none; JB: study support and speaker fees from Boston Scientific, Abbott, Medtronic; TL: modest speaker fees: Abbott, Boston Scientific, Lifetech; HS: reports study honoraria to institution, travel expenses, consulting fees $1<25.000 €$ : 4tech Cardio, Abbott, Ablative Solutions, Ancora Heart, Append Medical, Axon, Bavaria Medizin Technologie GmbH, Bioventrix, Boston Scientific, Carag, Cardiac Dimensions, Cardiac Success, Cardimed, Celonova, Contego, Croivalve, CVRx, Dinova, Edwards, Endobar, Endologix, Endomatic, Hangzhou Nuomao Medtech, Holistick Medical, Intershunt, K2, Lifetech, Magenta, Maquet Getinge Group, Medtronic,
Metavention, Mitralix, Mokita, NXT Biomedical, Occlutech, Recor, Renal Guard, Terumo, Trisol, Vascular Dynamics, Vectorious Medtech, Venus, Venock, Vivasure Medical, Vvital Biomed, HI: speaker fees Boston Scientific, Abbott; DT: lecture fees/honoraria from Bayer Vital, Boehringer Ingelheim Pharma, Bristol-Myers Squibb, Daiichi Sankyo, Medtronic, Pfizer Pharma, Sanofi-Aventis, St. Jude Medical, ZOLL CMS; MH, JS: unrestricted grant from Boston Scientific; PK: rresearch support from EU, BHF, Leducq Foundation, Medical Research Council (UK), and DZHK; AR: travel grants/lecture/consultant fees from Medtronik, Ablamap, Biosense webster, KODEX-EPD, Cardiofocus, Böhringer Ingelheim.

Ethics approval Ethics committee of the Rhineland-Palatinate State Medical Council (Landesärztekammer Rheinland-Pfalz) approved the study (837.173.14 (9412-F), 25.06.2014).

Consent for publication Given by all co-authors.

Open Access This article is licensed under a Creative Commons Attribution 4.0 International License, which permits use, sharing, adaptation, distribution and reproduction in any medium or format, as long as you give appropriate credit to the original author(s) and the source, provide a link to the Creative Commons licence, and indicate if changes were made. The images or other third party material in this article are included in the article's Creative Commons licence, unless indicated otherwise in a credit line to the material. If material is not included in the article's Creative Commons licence and your intended use is not permitted by statutory regulation or exceeds the permitted use, you will need to obtain permission directly from the copyright holder. To view a copy of this licence, visit http://creativecommons.org/licenses/by/4.0/.

\section{References}

1. Aminian A, Schmidt B, Mazzone P, Berti S, Fischer S, Montorfano M, Cheung CLS, Juha Lund M, Federico A, Gage R, CruzGonzalez I, Omran H, Tarantini G, Jens E-K (2019) Incidence, characterization, and clinical impact of device-related thrombus following left atrial appendage occlusion in the prospective global AMPLATZER amulet observational study. JACC Cardiovasc Interv 12:1003-1014

2. Boersma LV, Ince H, Kische S, Pokushalov E, Schmitz T, Schmidt B, Gori T, Meincke F, Protopopov AV, Betts T, Foley D, Sievert H, Mazzone P, De Potter T, Vireca E, Stein K, Bergmann MW (2017) Efficacy and safety of left atrial appendage closure with WATCHMAN in patients with or without contraindication to oral anticoagulation: 1-year follow-up outcome data of the EWOLUTION trial. Heart Rhythm 14:1302-1308

3. Brachmann J, Lewalter T, Akin I, Sievert H, Geist V, Zeymer U, Erkapic D, Mudra H, Pleger S, Hochadel M, Senges J (2020) Interventional occlusion of left atrial appendage in patients with atrial fibrillation Acute and long-term outcome of occluder implantation in the LAARGE Registry. J Interv Card Electrophysiol 58:273-280

4. Buist, T.J., Zipes, D.P. \& Elvan, A. Atrial fibrillation ablation strategies and technologies: past, present, and future. Clin Res Cardiol (2020). https://doi.org/10.1007/s00392-020-01751-5

5. Chen LY, Chung MK, Allen LA, Ezekowitz M, Furie KL, McCabe P, Noseworthy PA, Perez MV, Turakhia MP (2018) Atrial fibrillation burden: moving beyond atrial fibrillation as a binary entity: a scientific statement from the American heart association. Circulation 137:e623-e644 
6. Chugh SS, Havmoeller R, Narayanan K, Singh D, Rienstra M, Benjamin EJ, Gillum RF, Kim YH, McAnulty JH Jr, Zheng ZJ, Forouzanfar MH, Naghavi M, Mensah GA, Ezzati M, Murray CJ (2014) Worldwide epidemiology of atrial fibrillation: a Global Burden of Disease 2010 Study. Circulation 129:837-847

7. Biase Di, Luigi PS, Anselmino M, Mohanty P, Salvetti I, Gili S, Rodney Horton E, Javier S, Bai R, Mohanty S, Pump A, Brantes MC, Joseph Gallinghouse G, David Burkhardt J, Cesarani F, Scaglione M, Natale A, Gaita F (2012) Does the left atrial appendage morphology correlate with the risk of stroke in patients with atrial fibrillation? J Am Coll Cardiol 60:531-538

8. Dukkipati Srinivas R, Saibal Kar R, Holmes David K, Shephal D, Vijendra Swarup N, Douglas G, Brijeshwar Maini T, Gordon Nicole L, Michael M, Reddy Vivek Y (2018) Devicerelated thrombus after left atrial appendage closure. Circulation 138:874-885

9. Fastner C, Brachmann J, Lewalter T, Zeymer U, Sievert H, Borggrefe M, Nienaber CA, Weiss C, Pleger ST, Ince H, Maier J, Achenbach S, Sigusch HH, Hochadel M, Schneider S, Senges J, Akin I (2021) Left atrial appendage closure in patients with chronic kidney disease: results from the German multicentre LAARGE registry. Clin Res Cardiol 110:12-20

10. Friberg L, Tabrizi F, Englund A (2016) Catheter ablation for atrial fibrillation is associated with lower incidence of stroke and death: data from Swedish health registries. Eur Heart J 37:2478-2487

11. Ganesan AN, Chew DP, Hartshorne T, Selvanayagam JB, Aylward PE, Sanders P, McGavigan AD (2016) The impact of atrial fibrillation type on the risk of thromboembolism, mortality, and bleeding: a systematic review and meta-analysis. Eur Heart $\mathbf{J}$ 37:1591-1602

12. Glassy MS, Wung W, Westcott S, Smith TW, Fan D, Rogers JH, Singh GD (2019) Watchman occlusion in long-standing persistent atrial fibrillation. JACC Cardiovasc Interv 12:1018-1026

13. Goette A, Eckardt L, Valgimigli M, Lewalter T, Laeis P, Reimitz PE, Smolnik R, Zierhut W, Tijssen JG (2020) and Vranckx, P Clinical risk predictors in atrial fibrillation patients following successful coronary stenting: ENTRUST-AF PCI sub-analysis. Clin Res Cardiol https://doi.org/10.1007/s00392-020-01760-4

14. Goette A, Lip GYH, Jin J, Heidbuchel H, Cohen A-A, Ezekowitz M, Merino JL (2020) Differences in thromboembolic complications between paroxysmal and persistent atrial fibrillation patients following electrical cardioversion (from the ENSURE-AF study). Am J Cardiol 131:27-32

15. Hindricks G, Potpara T, Dagres N, Arbelo E, Bax JJ, BlomströmLundqvist C, Boriani G, Castella M, Dan G-A, Dilaveris PE, Fauchier L, Filippatos G, Kalman JM, Meir ML, Lane DA, Lebeau J-P, Lettino M, Lip GYH, Pinto FJ, Thomas GN, Valgimigli M, Van Gelder IC, Van Putte BP, Watkins CL, ESC Scientific Document Group (2021) ESC guidelines for the diagnosis and management of atrial fibrillation developed in collaboration with the European Association for Cardio-Thoracic Surgery (EACTS): the Task Force for the diagnosis and management of atrial fibrillation of the European Society of Cardiology (ESC) Developed with the special contribution of the European Heart Rhythm Association (EHRA) of the ESC. Eur Heart J 42(5):373-498. https://doi.org/ 10.1093/eurheartj/ehaa612

16. Hohnloser SH, Pajitnev D, Pogue J, Healey JS, Pfeffer MA, Yusuf S, Connolly SJ (2007) Incidence of stroke in paroxysmal versus sustained atrial fibrillation in patients taking oral anticoagulation or combined antiplatelet therapy: an ACTIVE W substudy. J Am Coll Cardiol 50:2156-2161
17. Kirchhof P, Camm AJ, Goette A, Brandes A, Eckardt L, Elvan A, Fetsch T, van Gelder IC, Haase D, Haegeli LM, Hamann F, Heidbuchel H, Hindricks G, Kautzner J, Kuck KH, Mont L, Ng GA, Rekosz J, Schoen N, Schotten U, Suling A, Taggeselle J, Themistoclakis S, Vettorazzi E, Vardas P, Wegscheider K, Willems S, Crijns H, Breithardt G, East-Afnet Trial Investigators (2020) Early rhythm-control therapy in patients with atrial fibrillation. N Engl J Med 383:1305-1316

18. Kishima H, Mine T, Takahashi S, Ashida K, Ishihara M, Masuyama T (2016) Morphologic remodeling of left atrial appendage in patients with atrial fibrillation. Heart Rhythm 13:1823-1828

19. Nührich JM, Kuck K-H, Andresen D, Steven D, Spitzer SG, Hoffmann E, Schumacher B, Eckardt L, Brachmann J, Lewalter T, Hochadel M, Senges J, Willems S, Hoffmann BA (2015) Oral anticoagulation is frequently discontinued after ablation of paroxysmal atrial fibrillation despite previous stroke: data from the German Ablation Registry. Clin Res Cardiol 104:463-470

20. Reddy Vivek Y, Doshi Shephal K, Saibal Kar N, Gibson Douglas J, Matthew P, Kenneth Huber P, Rodney H, Buchbinder M, Petr Neuzil T, Gordon Nicole R, David H (2017) 5-year outcomes after left atrial appendage closure. J Am Coll Cardiol 70:2964-2975

21. Rillig A, Bellmann B, Skurk C, Leistner DM, Haeusler KG, Lin T, Geran R, Koehler L, Guttmann S, Steffens D, Kasner M, Jakob P, Tscholl V, Roser M, Lenz K, Villringer K, Park J-W, Fiebach JB, Landmesser U (2018) Left atrial appendage angiography is associated with the incidence and number of magnetic resonance imaging-detected brain lesions after percutaneous catheter-based left atrial appendage closure. Heart Rhythm 15:3-8

22. Rottner L, Bellmann B, Lin T, Reissmann B, Tönnis T, Schleberger R, Nies M, Jungen C, Dinshaw L, Klatt N, Dickow J, Münkler P, Meyer C, Metzner A, Rillig A (2020) Catheter ablation of atrial fibrillation: state of the art and future perspectives. Cardiol Ther 9:45-58

23. Steinberg BA, Hellkamp AS, Lokhnygina Y, Patel MR, Breithardt G, Hankey GJ, Becker RC, Singer DE, Halperin JL, Hacke W, Nessel CC, Berkowitz SD, Mahaffey KW, Fox KAA, Califf RM, Piccini JP, on behalf of the ROCKET-AF Steering Committee, and Investigators (2014) Higher risk of death and stroke in patients with persistent vs. paroxysmal atrial fibrillation: results from the ROCKET-AF Trial. Eur Heart J 36:288-296

24. Apostolos T, Holmes DR Jr, Gafoor S, Ruiz CE, BlomströmLundqvist C, Diener H-C, Cappato R, Kar S, Lee RJ, Byrne RA, Ibrahim R, Lakkireddy D, Soliman OI, Nabauer M, Schneider S, Brachmann J, Saver JL, Tiemann K, Sievert H, Camm AJ, Lewalter T (2016) Percutaneous left atrial appendage occlusion: the Munich consensus document on definitions, endpoints, and data collection requirements for clinical studies. EP Europace 19:4-15

25. Wolf PA, Abbott RD, Kannel WB (1991) Atrial fibrillation as an independent risk factor for stroke: the Framingham Study. Stroke 22:983-988

26. Glikson Michael, Wolff Rafael, Hindricks Gerhard, John Mandrola A, Camm John, Lip Gregory Y H, Fauchier Laurent, Betts Tim R, Lewalter Thorsten, Saw Jacqueline, Tzikas Apostolos, Sternik Leonid, Nietlispach Fabian, Berti Sergio, Sievert Horst, Bertog Stefan, Meier Bernhard (2020) ESC Scientific Document Group, EHRA/EAPCI expertconsensus statement on catheterbased left atrial appendage occlusion - an update. EP Europace 22(2):184. https://doi.org/10.1093/europace/euz258 